\title{
Operant Conditioning of Head-Waving in Aplysia. I. Identified Muscles Involved in the Operant Response
}

\author{
David G. Cook and Thomas J. Carew \\ Departments of Psychology and Biology, Yale University, New Haven, Connecticut 06520
}

\begin{abstract}
A basic goal in the neurosciences is to understand the cellular mechanisms underlying associative learning. The 2 major forms of associative learning are classical conditioning and operant conditioning. In recent years considerable progress has been made towards a cellular analysis of classical conditioning in a number of different preparations. In contrast, the cellular mechanisms underlying operant conditioning are poorly understood. Since the marine mollusc Aplysia has proved to be a powerful preparation for studying cellular and molecular mechanisms of a variety of forms of learning, including classical conditioning, we asked whether Aplysia might also be capable of exhibiting operant conditioning. In previous experiments we found that a naturally occurring behavior, the head-waving response of Aplysia, could be operantly conditioned (Cook and Carew, 1986).

In the present paper we have carried out a quantitative analysis of the horizontal and vertical components of the head-waving response at the level of individual muscle groups. We have identified a discrete, bilateral band of neck muscles, the lateral columellar muscles (LCMs), whose electromyographic (EMG) activity is significantly correlated with the horizontal component of head-waving, the component modified by operant conditioning. Since head-waving is a complex behavioral response involving a variety of different muscle groups, using a restricted system such as the LCMs as an analog of the head-waving response will greatly facilitate a cellular analysis of operant conditioning in Aplysia.
\end{abstract}

In recent years, significant strides have been made in the cellular and molecular analysis of learning and memory. Progress has been especially rapid in a number of invertebrate preparations, which provide the advantage of a relatively simple CNS and the capacity to exhibit a wide array of learning phenomena (Carew and Sahley, 1986; Mpitsos and Lukowiak, 1986; Alkon, 1987; Byrne, 1987; Hawkins et al., 1987; Crow, 1988). Learning has often been divided into 2 principal classes, nonassociative and associative (Mackintosh, 1974; Rescorla, 1988). The 3 major forms of nonassociative learning-habituation, dishabituation, and sensitization - have each been studied extensively in behavioral and mechanistic terms, especially in the marine mol-

\footnotetext{
Received Dec. 8, 1988; revised Mar. 3, 1989; accepted Mar. 6, 1989.

We wish to thank Dorothy Shaffer and Russell Martin for their great assistance in collecting data, and Frederick Kuenzi and Mark Stopfer for their helpful criticism of the manuscript. This work was supported by NIH Grant 5-RO1-MH41083 and by NIH BRSG Grant 507-RR-075015 to T.J.C.

Correspondence should be addressed to Thomas J. Carew, Yale University Departments of Psychology and Biology, Yale Station 11A, New Haven, CT 06520.

Copyright (C) 1989 Society for Neuroscience $0270-6474 / 89 / 093097-10 \$ 02.00 / 0$
}

lusc Aplysia (Carew et al., 1971; Kandel and Schwartz, 1982; Walters et al., 1983a, b; Byrne, 1985; Hochner et al., 1986a, b; Marcus et al., 1988).

The 2 major forms of associative learning are classical conditioning and operant conditioning. Classical conditioning has received extensive attention in a number of invertebrate animals (Carew and Sahley, 1986; Byrne, 1987; Hawkins et al., 1987), and important insights into cellular and molecular mechanisms contributing to classical conditioning have been obtained, particularly in 2 preparations, Hermissenda (Farley and Alkon, 1982; Crow, 1985a, b, 1988; Alkon, 1987) and Aplysia (Carew et al., 1983; Hawkins et al., 1983; Walters and Byrne, 1983a, b). However, with the exception of early work by Hoyle and colleagues (Woolacott and Hoyle, 1977; Hoyle, 1982a, b), very little is known about the cellular mechanisms of operant conditioning. Thus, we were interested in examining whether $A p l y$ sia were also capable of exhibiting operant conditioning, with the goal of utilizing the advantages of Aplysia for a behavioral and cellular analysis of this major form of associative learning.

The behavior we have examined is the head-waving response of Aplysia. This is a naturally occurring behavior that can be evoked when the animal is suspended underwater above a substrate. Under these conditions the animal will sweep its head from side to side for up to an hour; presumably searching for a substrate to attach to. Aspects of this response are also observed in a number of other behavioral contexts in Aplysia, including egg-laying (Cobbs and Pinsker, 1982a, b; Ferguson et al., 1989a, b), locomotion (Jahan-Parwar and Fredman, 1978a, b; Hening et al., 1979; Fredman and Jahan-Parwar, 1983), and feeding (Preston and Lee, 1973; Kupfermann, 1974a, b; Bablanian et al., 1987). We found that the head-waving response of Aplysia could be operantly conditioned readily by the contingent presentation of a punishing stimulus, bright light, which the animals find aversive. The operant learning is rapidly acquired (within $10 \mathrm{~min}$ ) and is critically dependent upon contingent reinforcement since yoked-control animals do not learn (Cook and Carew, 1986).

In the present series of papers we begin a cellular analysis of operant conditioning in Aplysia. Such an analysis requires investigating both the motor output pathways responsible for production of the operant response (Cook and Carew, 1988, 1989a), and the reinforcement pathways responsible for the contingent modification of the operant response (Cook and Carew, 1989b). In the present paper we have identified a number of behavioral components of the head-waving response. The horizontal (sideto-side) component is the major component of head-waving modified by operant conditioning (Cook and Carew, 1986). Moreover, we have identified a discrete, bilateral band of neck muscles, the lateral columellar muscles (LCMs), whose activity is significantly correlated with the horizontal component of the 
behavioral response. In the second paper we report that, in addition to intact head-waving behavior, the electromyographic (EMG) activity of the LCMs themselves can be modified by contingent reinforcement, thus providing a simplified experimental system for detailed cellular studies of operant conditioning. Finally, in the third paper, we describe a cellular analysis of 2 candidate reinforcement pathways that may contribute to operant conditioning of head-waving in Aplysia.

Some of the results in the present paper have been presented in abstract form (Cook and Carew, 1988).

\section{Materials and Methods}

Animals. Eight adult Aplysia californica (150-250 gm), supplied by Sealife Supply (Sand City, CA), were used in this study. The animals were individually housed in a 1140 liter aquarium filled with circulating aerated artificial seawater (Instant Ocean; Mentor, $\mathrm{OH}$ ) and maintained at room temperature $\left(20-22^{\circ} \mathrm{C}\right)$.

Muscle cuff electrodes. The EMG cuff electrodes were constructed using the methods of Pinsker and Eberly (1982). The cuffs were made of 0.025 inch (i.d.) silastic tubing (Atlantic Healthcare Products, Andover, MA) approximately $3-5 \mathrm{~mm}$ in length. The cuf was split lengthwise on one side so that it could be slipped around the muscle (see below). A small $(1 \mathrm{~mm})$ segment of bare 0.001 inch stress-relieved stainless steel wire (California Fine Wire; Grover City, CA) was secured along the inner surface of the cuff to make electrical contact with the muscle. The external lead from the cuff (made from the same stainless steel wire) was approximately $30 \mathrm{~cm}$ in length and terminated at the preamplifier head-stage. For additional strength and insulation, the external lead was coated with a thin layer of dental impression material (Syborn/Kerr, Romulus, MI). Recordings from muscle cuffs were obtained using single-ended amplification.

Surgery. To surgically implant the cuff electrodes animals were first anesthetized by injecting an amount of isotonic $\mathrm{MgCl}_{2}$ solution equal to $50 \%$ of their body weight into the body cavity. Once anesthetized, the animals were placed on their sides in a bath of high- $\mathrm{Mg}^{2+}(210 \mathrm{~mm})$ Aplysia Ringer's solution. During surgery, which typically lasted $1 \mathrm{hr}$, the animals were draped with a damp light-opaque cover that kept them from being exposed to the bright fiberoptic operating lights (Dolan Jenner, Woburn, MA)

A small incision was made near the base of the neck directly over the LCM. The superficial layers of diffuse muscle were retracted, taking care to avoid damage to nerves in the region of the dissection. Once the LCM was exposed, a band of the muscle that could fit snugly into the cuff electrode was teased away from the rest of the LCM so that the cuff could be slipped around it. The cuff was then secured to the muscle band with a loop of suture around each end of the cuff. Next the external lead was passed through the incision and sutured to the body wall about 10-15 mm away from the cuff in order to reduce stress on the cuff and the muscle. The incision was sutured closed, and the procedure was repeated on the other side of the animal. The animals were returned to their home tank and allowed to recover for $24 \mathrm{hr}$ prior to testing. After implantation of the cuff electrode, the LCM muscles appeared to contract normally, both in response to body wall stimulation and during head-waving movements. Similar applications of this technique (Pinsker and Eberly, 1982; Bablanian et al., 1987) also appear to produce little or no impairment of nerve or muscle function.

Apparatus. The testing chamber was a $100 \times 100 \times 100 \mathrm{~cm}$ clear Plexiglas aquarium filled with Instant Ocean at room temperature (20$22^{\circ} \mathrm{C}$ ). The chamber was housed in a Faraday cage that provided electrical shielding but still allowed an unobstructed view of the behaving animal (Fig. 1). The animals were suspended underwater in the chamber so they could not touch the bottom or sides of the tank. Suspension was accomplished with small stainless steel hooks inserted bilaterally into the parapodia. The hooks were attached to suture lines that were secured to a Plexiglas rod positioned over the testing tank. In the suspended position animals would head-wave actively for up to an hour. The electrode leads were wound around the suspension lines and plugged into preamplifier head-stages which were located over the testing tank.

EMG signals were amplified by Grass P511 AC amplifiers (Quincy, MA) and recorded on an FM instrumentation recorder (Vetter, Rebersberg, PA). In addition, the amplified EMG signals were processed by voltage-level window discriminators (Frederick Haer, Brunswick, ME). Thus, it was possible to reject all subthreshold electrical noise, as well as movement artifacts (which were usually substantially larger than the EMG signals). The window discriminators generated a TTL level pulse for each muscle action potential; each pulse from both left and right EMG channels was then counted, displayed, and stored on a computer (IBM PC/AT, with Data Translation 2821-F-8/DI data-acquisition system; Data Translation, Marlboro, MA) over a $600 \mathrm{sec}$ testing period. The computer provided on-line computation of both differential (left - right spikes/sec) and summed (left + right spikes/sec) bilateral EMG activity.

Quantification of head-waving behavior. Two aspects of head-waving behavior were observed and quantified: movements in the horizontal plane (side to side) and movements in the vertical plane (up and down). Quantification was carried out by an observer who operated a joystick which was used to track the magnitude and direction of the animals' movements in both planes. The observer tracked the animals' headwaving movements with reference to horizontal and vertical arrays of light emitting diodes (LEDs) placed over the tank so that the observer could easily attend to both the animals' movements and the LED display (Fig. 1). The horizontal and vertical outputs of the joystick were digitized, displayed, and stored by the computer; this permitted on-line comparisons of the horizontal and vertical movements of the animal with the left and right EMG activity of the LCMs.

Video analysis. A video analysis was performed to validate the method of quantifying head-waving behavior by means of a joystick. This analysis was accomplished by making simultaneous video recordings with 2 cameras of an animal head-waving. One camera was placed directly above the testing chamber facing down on the animal; the second camera was positioned to one side of the animal (providing an orthogonal view relative to the first overhead camera). Additionally, the LED array, indicating an observer's assessment of head-waving was videotaped in synchrony with the other 2 video recordings of the animal. Videotapes of head-waving behavior were analyzed by an observer who was blind to the LED scores from the joystick. The head-waving position on the videotape was determined by stop-frame analysis at $1 \mathrm{sec}$ intervals. For each $1 \mathrm{sec}$ sample the angle of neck flexion was quantified by tracing it on an acetate sheet placed on the video monitor. The magnitude of the neck flexion was then quantified by means of a computerized bit pad analysis (R. M. Biometrics, Nashville, TN) of the traced angles. Finally, the videotaped behavioral scores of horizontal and vertical head-waving were correlated with the scores derived from the joystick output produced by the observer.

Data analysis. All statistical analyses were performed on EMG and behavioral data that had been summed into 2 sec time bins. Standard procedures were used to perform analysis of variance, multiple regression, correlations, and cross-correlations (Minitab Software, State College, PA). In cases where $r$ coefficients for multiple animals were pooled, the $r$ values were first transformed to $z$ scores. Then, the average $z$ scores were reexpressed as average $r$ values (see Horowitz, 1974).

\section{Results}

The central question addressed in this study was whether the activity of an identified band of muscles, the LCMs, could be related predictably to the head-waving behavior of Aplysia. To examine this question it was first necessary to characterize the component movements involved in head-waving and to develop reliable methods for quantifying both the direction and magnitude of the head-waving response.

\section{Components of head-waving behavior}

Head-waving in Aplysia can be divided into 4 principal components: (1) horizontal (side-to-side) movements; (2) vertical (up-and-down) movements; (3) extension/contraction of the head; and (4) a rotary movement of the head and neck around the longitudinal axis of the animal. Although the animal exhibits a wide variety of combinations of these 4 types of movements, horizontal and vertical movements are by far the most commonly observed aspects of head-waving. The horizontal movements were of particular interest because in previous behavioral experiments it was head-waving in the horizontal plane that was modified by operant conditioning (Cook and Carew, 1986). By 


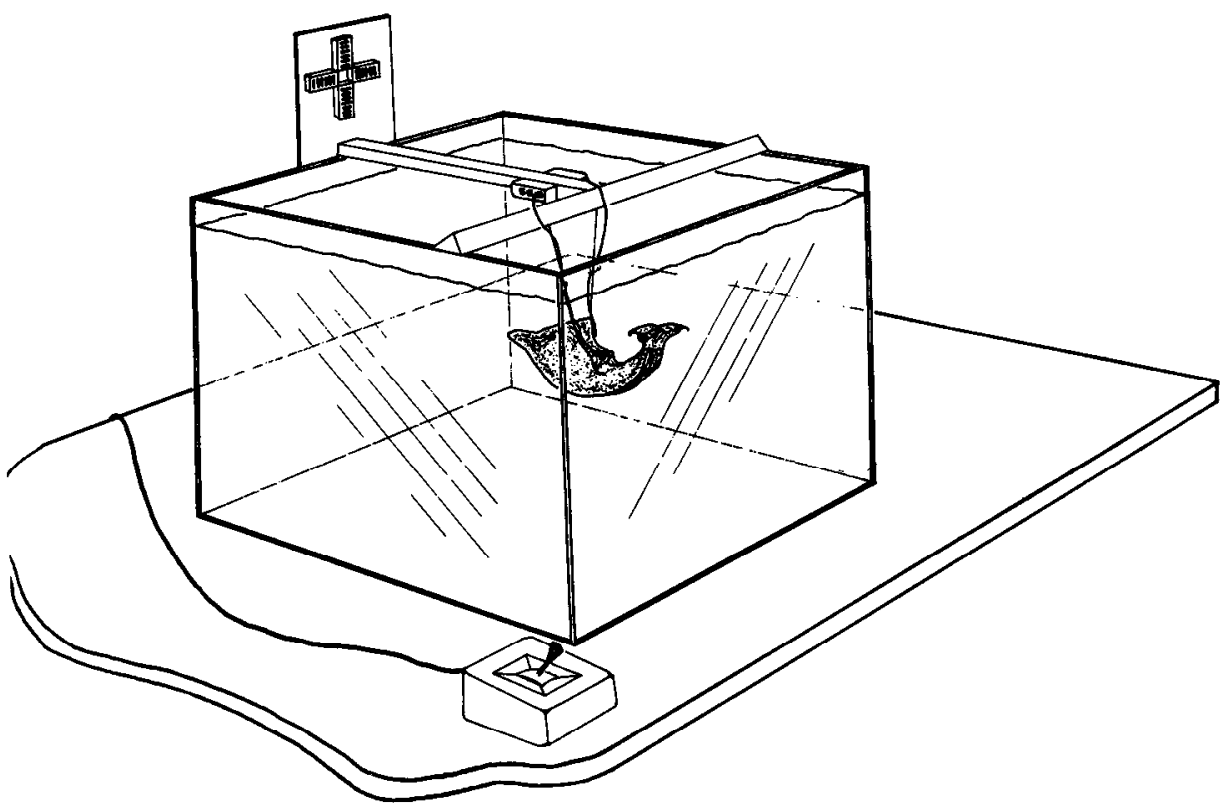

Figure 1. Behavioral testing chamber. Animals are suspended underwater, but above the substrate, by means of small parapodial hooks attached to suture lines secured to an overhead Plexiglas rod. External leads from implanted cuff electrodes (see Fig. 4) are wound around the suspension lines and inserted into overhead preamplifier head-stages. A computer joystick (bottom left) is used by an observer to track the head-waving response, both in magnitude and direction. Head-waving movements are tracked with reference to an intersecting horizontal and vertical array of 20 LEDs positioned over the tank. The joystick signals are digitized by a computer that simultaneously records EMG spike rate from the LCMs. This system permitted on-line correlations of head-waving behavior and EMG activity (see Materials and Methods for details). contrast, the 2 remaining components, contraction/extension and rotary movements, occurred so rarely under the conditions in which we observed head-waving (animals exhibited these components less than $5 \%$ of the observation time) that they contributed very little to the overall behavior. Thus, in the present study, we restricted our analysis to movements in the horizontal and vertical planes.

\section{Quantification of head-waving behavior}

In order to relate head-waving to the activity of the LCMs, it was first necessary to develop a reliable method for quantifying the movements of Aplysia in the horizontal and vertical plancs. To accomplish this, an observer using a joystick (Fig. 1) visually monitored and tracked the angle subtended by the base of the neck with respect to the orientation of the center of the body
(Fig. 2). Since the observer could constantly view the animal both from the side and from above, any angular changes of the body with respect to the attachment site on the parapodia could be compensated for by altering the viewing position during the observation period. For most animals the full range of possible horizontal movement from left to right was approximately $270^{\circ}$ $\left(135^{\circ}\right.$ side), and the range for vertical movement was approximately $200^{\circ}\left(100^{\circ}\right.$ up and $100^{\circ}$ down). Both planes of movement were subdivided into 20 even increments $(13.5 \%$ unit in the horizontal and $10 \%$ unit in the vertical plane). Thus, as illustrated in Figure 2, a horizontal head movement to the right side of the body of $50^{\circ}$ would fall in the +4 range $\left(40.5^{\circ}-54^{\circ}\right)$. This method of quantifying and recording the behavior has several advantages: (1) It is noninvasive and does not involve connecting transducers to the animal that might restrict or alter its
A

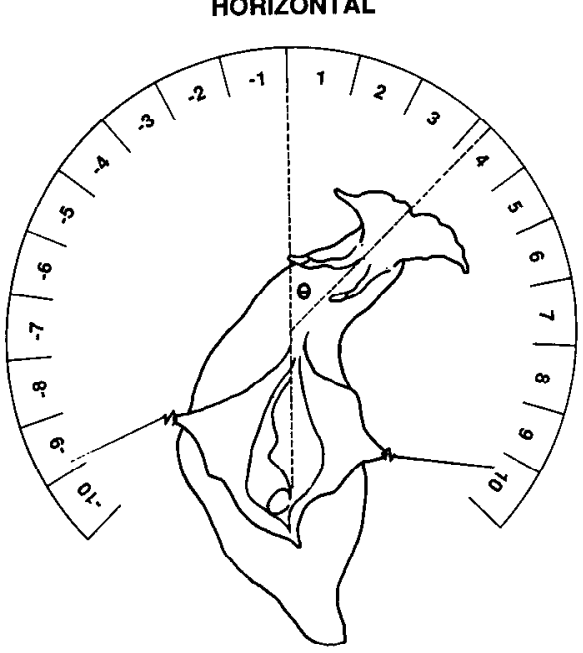

B

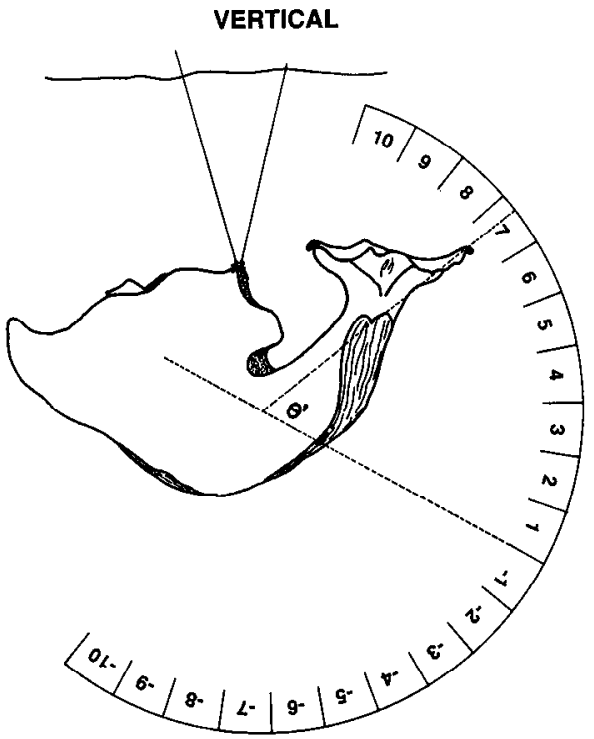

Figure 2. Quantification of headwaving. $A$, To measure head-waving in the horizontal plane an observer visually monitored the angle $(\theta)$ subtended by the base of the neck, with respect to the orientation of the center of the body (dashed lines). The full range of horizontal head-waving was approximately $270^{\circ}\left(135^{\circ} /\right.$ side $)$. This plane of movement was divided into 20 even increments $(13.5 \%$ unit), 10 increments on each side of the midline. $B$, For vertical head-waving, the angle $\left(\theta^{\prime}\right)$ was determined as in $A$. The full range of vertical movement was approximately $100^{\circ}$ above and $100^{\circ}$ below the horizontal meridian. This plane of movement was divided into 20 even increments $\left(10^{\circ}\right.$ per unit), 10 increments on either side of the horizontal plane. 
A

Figure 3. Correlation between 2 measures of head-waving. Scatter plots show a highly significant correlation, in both horizontal and vertical planes, between observer-derived measures of headwaving (using a joystick to track the behavior) and an objective stop-frame video analysis carried out simultaneously (using blind procedures).

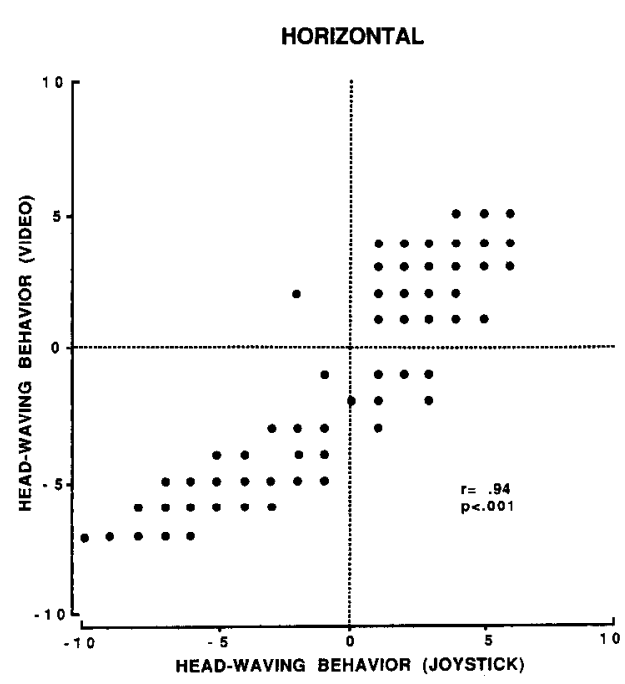

B

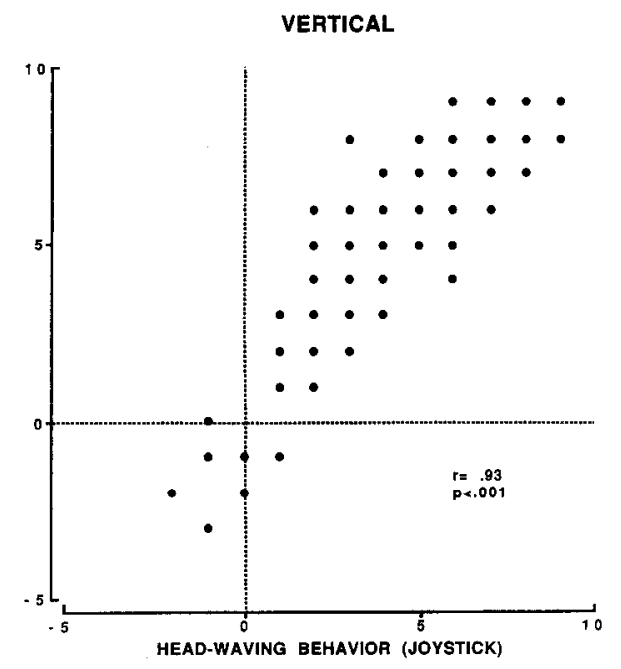

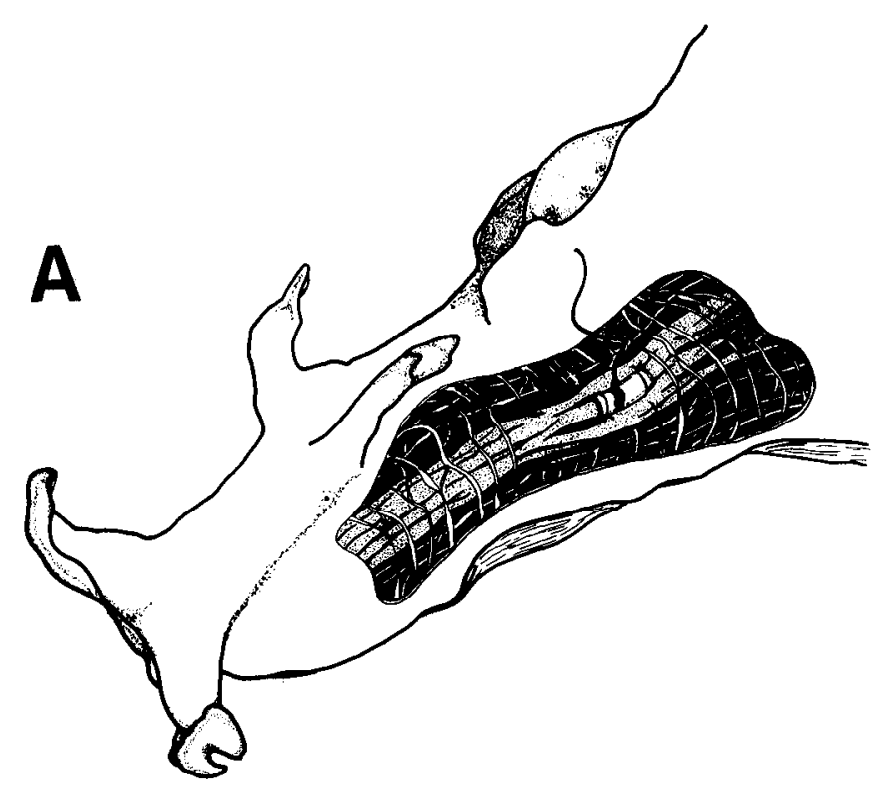

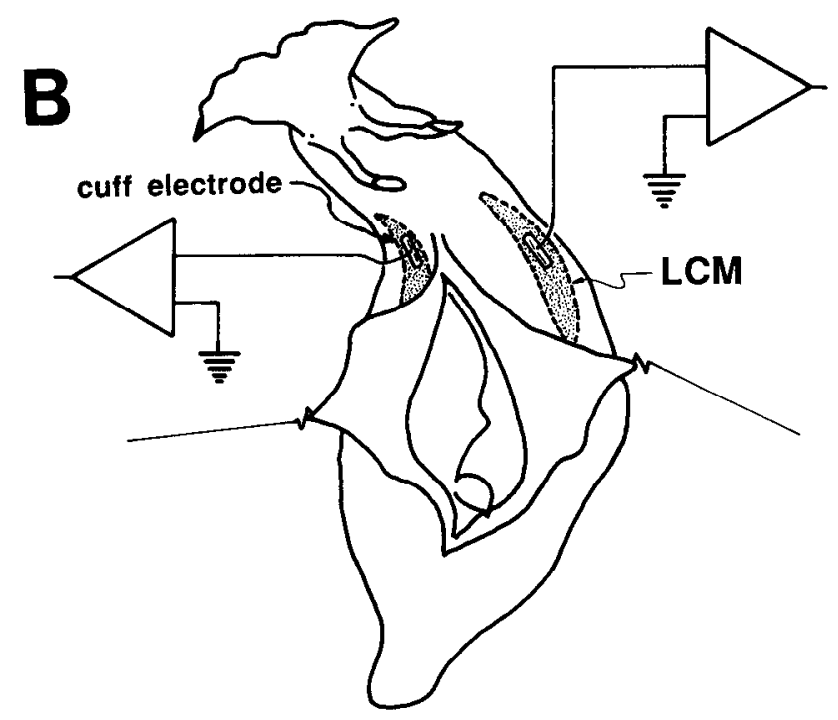

movements; (2) it is relatively simple to implement; and (3) it can be carried out in real time, rather than in a postexperimental analysis.

At the outset, it was important to assure that the joystick movements registered by an observer accurately reflected the actual head-waving behavior of the animals. To examine this, we conducted a test in which the head-waving behavior of an animal was videotaped with 2 cameras: one from above and the other from one side (see Materials and Methods). The behavior was also simultaneously assessed by an observer using a joystick to track the direction and magnitude of both the horizontal and vertical movements of head-waving. The LED display from the joystick was videotaped along with the behavior. We next carried out a stop-frame analysis of the videotape recordings (at $1 \mathrm{sec}$ increments) assessing the exact position of the animal at 300 successive points in time. We then determined the correlation between head-waving position derived from the video analysis and head-waving position derived from the output of the joystick. The results are illustrated in Figure 3, which shows a scatter plot of head-waving behavior scores obtained from the joystick output and the video analysis for both the horizontal and vertical planes. The correlation between the 2 measures of horizontal head-waving was highly significant ( $r=$ $0.94, p<0.001 ; F[1,298]=2177$; Fig. $3 A)$. The measures of vertical head-waving were also highly significant $(r=0.93, p<$ $0.001 ; F[1,274]=1695$; Fig. $3 B$ ). Moreover, a linear-regression analysis revealed that the slope of the regression lines were very close to unity for both the horizontal (slope $=0.93$ ) and vertical scores (slope $=1.06$ ), indicating that the scale of the relationship between the joystick and video-derived scores of head-waving and behavior were very close to $1: 1$.

In summary, the results described above show that the method of quantifying head-waving with the joystick, which codes the magnitude and direction of the movement, provides a non-

\footnotetext{
Figure 4. Lateral columellar muscles. $A$, Schematic illustration of a left LCM extending laterally in the body wall along the neck. A muscle cuff electrode is shown implanted around a fascicle of the muscle band. $B$, Diagram of the bilateral placement of the LCMs.
} 


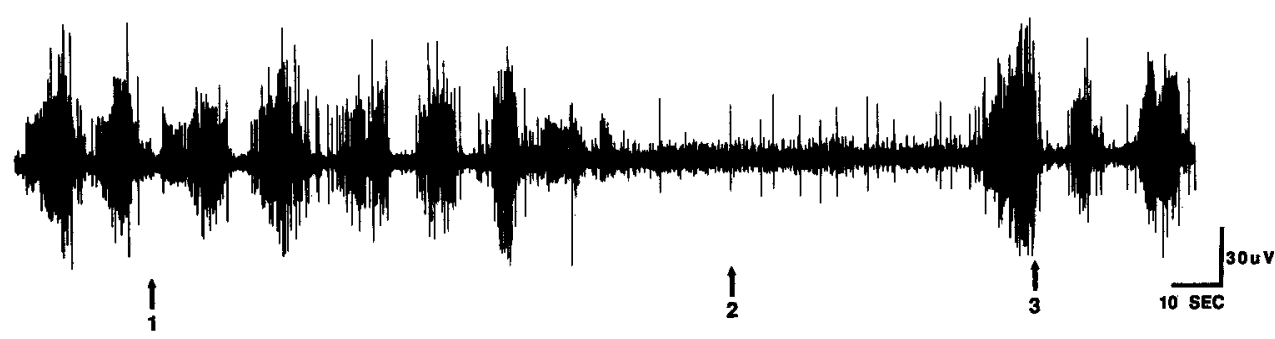

$\mathbf{B}_{1}$

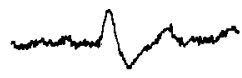

$\mathbf{B}_{2}$

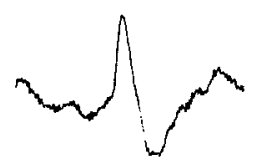

$\mathbf{B}_{3}$

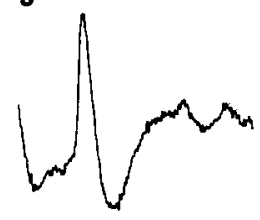

Figure 5. EMG recordings from an LCM in a freely behaving animal. $A, \mathrm{~A}$ prolonged record $(250 \mathrm{sec})$ showing typical EMG activity from an LCM during head-waving. Note the varied amplitudes of muscle action potentials. $B$, Expanded sweep speed to illustrate individual action potentials in $A\left(B_{1}-B_{s}\right.$ are taken from portions of record indicated by arrows in $A$ ). Although the amplitude of different muscle action potentials varied considerably, their duration (120-150 msec) and waveform were quite uniform. invasive and highly accurate representation of head-waving in the horizontal and vertical planes.

\section{The LCMS}

Based upon their anatomical location, the LCMs are likely to play an important role in head-waving. These muscle bands extend bilaterally and longitudinally along the lateral aspect of the neck and head region of the body wall in Aplysia (Fig. 4A). The LCMs were additionally attractive for study because of their discrete, banded structure, which facilitated reliable bilateral implantation of the EMG cuff electrodes (Fig. $4 B$; see Materials and Methods).

An example of a typical EMG recording from a muscle cuff electrode in a freely behaving animal is provided in Figure $5 \mathrm{~A}$, which shows a $250 \mathrm{sec}$ recording from an LCM. Typically, recordings were comprised of multiple EMG potentials that varied considerably in amplitude, (Fig $5 B_{1-3}$ ). However, despite their difference in size, the EMG action potentials were quite uniform in both duration (150-200 msec) and waveform (triphasic in nature). These characteristics allowed us to distinguish readily between EMG action potentials and occasional movement artifacts that were much larger in amplitude, much slower in duration, and not usually triphasic.

\section{Correlation between LCM activity and head-waving behavior}

Having established that we could quantitatively assess headwaving behavior and could reliably record from the LCMs in freely behaving animals, we next investigated the relationship between LCM activity and the horizontal head-waving response. Bilateral recordings were obtained from 8 animals while they head-waved during an 8-10 min observation period in a recording chamber (Fig. 1). Simultaneously, an observer (who could not see the EMG output of the LCMs) used the joystick to record the horizontal and vertical components of the headwaving behavior of the animals. The output of the joystick and the 2 EMG channels (from left and right LCMs) were input to a computer that compiled the EMG spike rate for each channel and the behavioral record every $2 \mathrm{sec}$ (see Materials and Methods).
An example of the relationship between head-waving behavior in the horizontal plane and EMG recordings from the left and right LCM recordings is shown in Figure $6 \mathrm{~A}$. There was a clear correspondence between EMG recordings and head-waving position. Specifically, at each point in the recording where the activity of the left LCM exceeded that of the right LCM; the animal's head was moving to the left. Conversely, at each point in time where activity in the right LCM exceeded the left, the animal's head was moving to the right. Thus, there is a clear relationship between differential (left - right) activity in the LCMs and horizontal head-waving behavior. A more fine-grained analysis of this relationship is shown in Figure $6 \mathrm{~B}$, where the differential EMG spike rate for a $100 \mathrm{sec}$ sample period (from the record shown in Fig. 6A) is plotted on an expanded time scale. Horizontal head-waving of the animal is plotted on the same time base. The differential LCM EMG activity closely maps onto horizontal head-waving of the animal. Moreover, by plotting the results in this fashion it is apparent that the behavioral response lagged differential LCM activity by approximately $6 \mathrm{sec}$ (see below).

To quantify the relationship between EMG and head-waving behavior, we analyzed the correlation between differential LCM activity and horizontal head-waving. In order to more accurately determine the correlation between these 2 variables, the behavioral data was phase-advanced $6 \mathrm{sec}$ with respect to the EMG data to compensate for the phase delay described above. The results from the animal shown in Figure 6 are illustrated in Figure 7, which shows a scatter plot of differential (left - right) EMG (on the abscissa) and head-waving position (on the ordinate). There was a highly significant correlation between the 2 measures $(r-0.74, F[1,265]-324.84, p<0.001)$. This analysis was repeated for each animal $(n=8)$. The individual correlations for each animal and the corresponding statistical probabilities are shown in Table 1. These results indicate that, for each animal, the correlation between the horizontal component of head-waving behavior and differential (left - right) EMG activity was highly significant. Thus, these data show that the electromyographic activity in the LCMs can serve as a reliable monitor of the head-waving response. 
Figure 6. Correlation between LCM activity and head-waving behavior. $A$ Top trace is the output of a joystick used by an observer to track head-waving behavior (dotted line is midline; excursions above and below the dotted line represent head-waving to the left and right, respectively). Bottom pair of traces are EMG recordings from the left and right LCM. Note that whenever activity in the left LCM exceeded the right, the animal was head-waving to the left; whenever the right EMG exceeded the left, the animal was head-waving to the right. $B$, Data from an expanded $(100$ sec) portion of the record in $A$ (segment sampled is shown by the bracketing vertical lines). Data are expressed as (1) differential EMG (left - right) on the left ordinate (dotted line), and (2) headwaving on the right ordinate (solid line). The midline is indicated by dashed horizontal line originating at the zero intercept. Whenever left EMG exceeded the right, or head-waving was to the left, data points fall above the horizontal line; whenever right EMG exceeded the left, or head-waving was to the right, data points fall below the line. There is a close correspondence between differential EMG and the headwaving of the animal (note that the behavioral response consistently lags EMG activity).

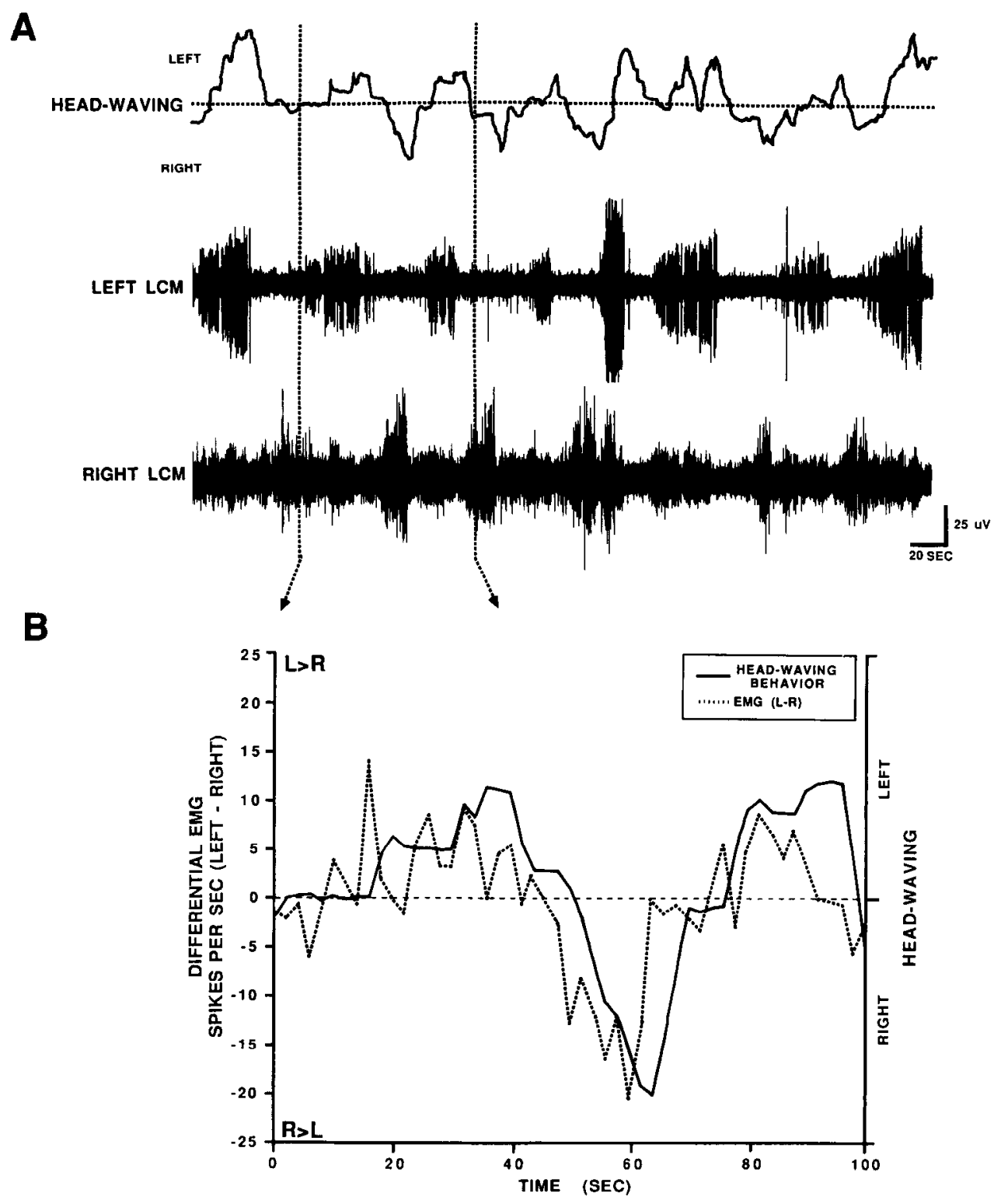

the results shown in the inset of Figure 8, which illustrates that in 5 of 8 animals, maximal cross-correlation coefficients were obtained at a phase lag of $6 \mathrm{sec}$. In addition, the results shown in Figure 8 illustrate that the average correlation between LCM activity and horizontal head movements for all animals in the study $(n=8)$ was quite high: $r=0.595$ ( \pm 0.09$)$.

\section{Head-waving in the vertical plane}

As mentioned earlier, the predominant component of headwaving we observed is a side-to-side sweeping movement of the head in the horizontal plane. Another frequently observed component of the response is head-waving in the vertical plane. During vertical head-waving, we found that, although animals were quite capable of downward movements of their heads (below the horizontal plane), the majority of vertical head movements were in the upward direction (above the horizontal plane). Given the bilateral anatomical arrangement of the LCMs in the sides of the body wall (Fig. 4), we expected that these muscle bands might be more or less synchronously active during vertical mately 6 sec. The reliability of this finding is emphasized by 


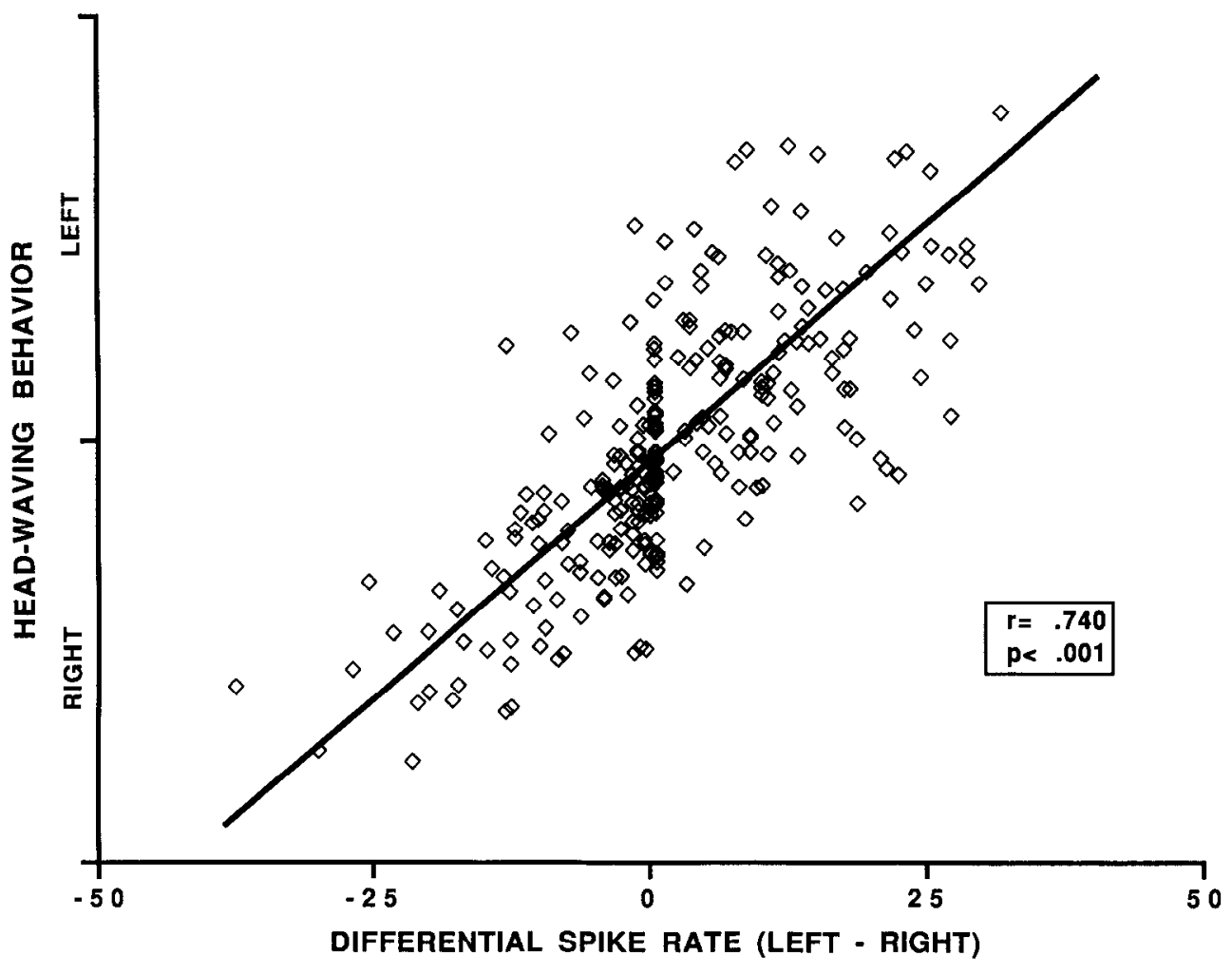

Figure 7. Scatter plot illustrating the relationship between LCM activity and the horizontal head-waving response. Data are from the animal whose records are shown in Figure 6. Differential EMG is plotted on the abscissa, head-waving position on the ordinate (behavioral data were phase advanced by $6 \mathrm{sec}$ with respect to the EMG data; see text). There is a highly significant correlation $(p<$ 0.001 ) between the 2 medsures. head lifts (in contrast to being differentially active as they are during horizontal head-waving). In most instances, this turned out to be the case: In 6 of the 8 animals there was a significant correlation between bilaterally synchronous LCM activity and vertical head-waving ( $p<0.001$ in each case). Nonetheless, the average correlation between synchronous (left + right) LCM activity and vertical head-waving $\left(r_{\text {vertical }}=0.345, \pm 0.10\right)$ was significantly lower than the correlation between differential (left - right) LCM activity and horizontal head-waving $\left(r_{\text {horizontal }}=\right.$ $0.595, \pm 0.09 ; t[14]=2.41, p<0.03)$. The lower correlation between vertical head movements and LCM activity is consistent with the interpretation that the LCMs are better suited for mediating side-to-side movements than for mediating headlifting movements.

In summary, our results warrant 2 conclusions: (1) during horizontal head-waving, the differential activity of the left and right LCMs is, on average, highly correlated with the behavioral response, and (2) during vertical head-waving, the synchronous activity of the left and right LCMs is usually, but not invariably, correlated with the behavioral response.

\section{Discussion}

Head-waving response in Aplysia

Head-waving movements in Aplysia occur under a wide variety of circumstances in the natural environment. For example, one prominent feature of egg-laying behavior in Aplysia is the use of head-waving to aid in egg-mass extrusion and in securing the eggs to a substrate (Kupfermann, 1970; Cobbs and Pinsker, $1982 \mathrm{a}, \mathrm{b})$. Rhythmic movements of the head and neck are also integral components of locomotion in Aplysia (Hening et al., 1979; Fredman and Jahan-Parwar, 1983). In addition, Aplysia actively head-wave in their search for food, and use a variety of complex head movements during tactile manipulations of food prior to its ingestion (Preston and Lee, 1973; Kupfermann, 1974a, b; Kupfermann and Carew, 1974; Bablanian et al., 1987). A reliable replica of the head-waving seen in the natural environment can be produced in the laboratory by suspending the animals above a substrate, which gives rise to vigorous headwaving for extended periods of time (Cook and Carew, 1986). Head-waving is a flexible behavior that comprises a number of components that are expressed to differing degrees in different behavioral contexts. For example, during egg-laying behavior, 4 discrete components of head-waving have been described, including (1) tamping (the head is used to compress the egg mass onto a substrate), (2) waving (side-to-side movements of the head and neck), (3) undulations (a combination of waving and head-lifting), and (4) weaving (a combination of waving and rhythmic contraction and extension of the neck) (Ferguson et al., 1989a).

In our current analysis of head-waving, in which Aplysia are suspended underwater above a substrate, we have found that

\begin{tabular}{|c|c|c|}
\hline $\mathrm{S}$ & $r$ & $\begin{array}{l}\text { Probability } \\
(p)\end{array}$ \\
\hline 1 & 0.706 & $<0.001$ \\
\hline 2 & 0.616 & $<0.001$ \\
\hline 3 & 0.247 & $<0.01$ \\
\hline 4 & 0.454 & $<0.001$ \\
\hline 5 & 0.476 & $<0.001$ \\
\hline 6 & 0.742 & $<0.001$ \\
\hline 7 & 0.602 & $<0.001$ \\
\hline 8 & 0.753 & $<0.001$ \\
\hline
\end{tabular}


Figure 8. Analysis of phase lag between differential LCM activity and head-waving. A cross-correlation analysis shows that the correlation between differential EMG from the LCMs and head-waving behavior, is maximal where behavior lags EMG by $6 \mathrm{sec}$. Inset, Distribution of individual peak cross-correlations for all animals (5 of 8 animals showed peak correlations at $6 \mathrm{sec}$ phase lag).

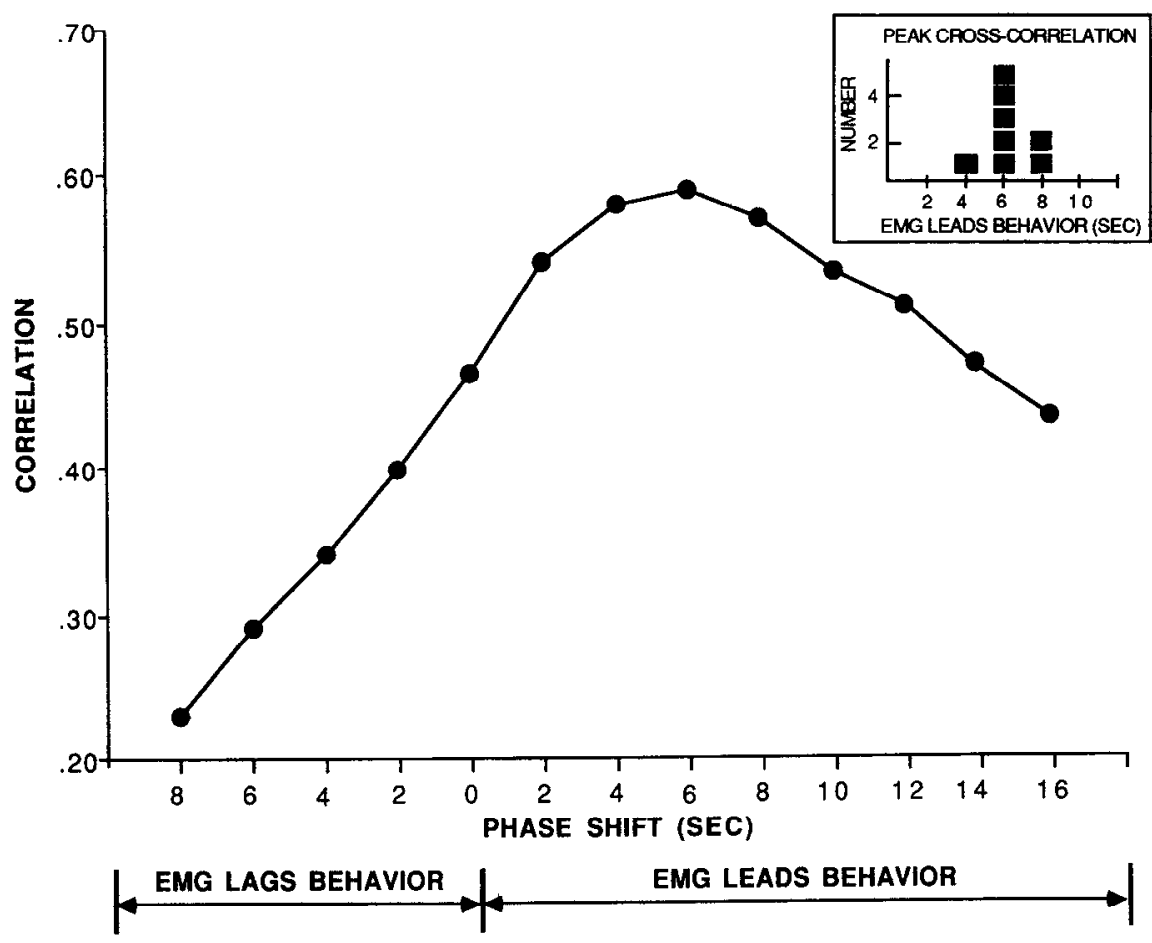

their head-waving behavior can be divided into 4 primary components: (1) horizontal head-waving (side-to-side movements of the head and neck), (2) vertical head-waving (up and down movements); (3) contraction and extension of the neck; and (4) a rotary motion of the head around the longitudinal axis of the animal. In our studies, the horizontal and vertical movements were the 2 major components of head-waving most often exhibited. Of the 2 major components, the horizontal component was by far the most frequently observed. Moreover, it is the horizontal component of the head-waving response that we operantly conditioned in previous behavioral experiments (Cook and Carew, 1986). Thus, in the present paper, we focused our attention primarily on head-waving in the horizontal plane.

\section{The LCMS}

The LCMs are prominent bilaterally symmetric muscle bands that run longitudinally in the lateral body wall. They originate in the head region, extend through the neck, and terminate in diffuse ramifications in the body wall in the posterior third of the animal (Brace, 1977a, b; Kuenzi and Carew, 1988). Thus, on anatomical grounds, these muscles appear well suited to contribute to the head-waving response since their reciprocal contraction and relaxation would give rise to lateral bending. In addition to the LCMs, there are other prominent muscle bands that also could contribute to head-waving behavior. These muscle groups include the dorsal longitudinal muscles (DLMs), the longitudinal foot muscles (LFMs), and the transversc muscles of the dorsal body wall. [For a detailed description of the anatomy of the molluscan body wall, see Brace (1977a, b).] The activity of some of these muscle groups has already been investigated in relationship to other behaviors in Aplysia. For example, contractions of the transverse muscles of the body wall are thought to cause neck extension, while the LFMs are active primarily during locomotion (Ferguson et al., 1989a, b). Finally, the DLMs show increased phasic excitation and inhibition dur- ing both head-waving and head undulations associated with feeding behavior (Bablanian et al., 1987).

In this paper we focused our attention exclusively on the LCMs. We have shown that in all animals studied, differential EMG activity in the LCMs was significantly correlated with side-to-side head-waving movements in the horizontal plane. Activity in the LCMs can also correlate with vertical head movements, although less well than with movements in the horizontal plane. The LCM correlation with vertical movements appears to be primarily associated with lifting of the head in conjunction with horizontal movements. These observations are consistent with the results of Bablanian and colleagues (1987), who reported that activity in nerves innervating the LCMs is occasionally observed during head lifting.

In summary, because of their large size and anatomical location, the LCMs are well suited to play an important role in horizontal head-waving. Moreover, their differential electrical activity during active head-waving correlates highly with the horizontal component of the overall behavioral response. Thus, although other muscle groups (including the DLMs and LFMs) are also likely to contribute to the head-waving behavior we observe, the activity of the LCMs alone can serve as a significant predictor of head-waving behavior in the horizontal plane.

\section{Phase lag between EMG and head-waving behavior}

In experiments in which we recorded EMG from the LCMs during active head-waving we found that overt head-waving behavior consistently lagged differential EMG activity in the LCMs by 5-6 sec. A phase lag of this magnitude is consistent with a previous report by Hening and colleagues (1979), who found latencies of 4-8 sec between the firing of motor neurons to the neck and the peak tension of neck muscles. Moreover, such long latencies do not appear to be restricted to movements of the head and neck in Aplysia. Similar long delays between the firing of peripheral siphon motor neurons and siphon with- 
drawal responses have also been observed (Bailey et al., 1979). However, long-latency behavioral responses are not the only behavioral options available to Aplysia. For example, measurable contractions of the gill in response to activation of single gill motor neurons can occur within $1 \mathrm{sec}$ (Kupfermann et al., 1974). In addition, Aplysia are capable of detecting a food stimulus delivered near the mouth and then completing the appropriate orienting response in less than 3 scc (Teyke and Kupfermann, 1988).

The reason for the relatively long delays in response systems such as the head-waving system of Aplysia between motor neuron activation or muscle activity, on the one hand, and overt behavior, on the other, is not clear. These long latencies may reflect, at least in part, inherent bioelastic properties of the neck muscles and body wall of Aplysia. Moreover, the hoemocel of Aplysia may also affect the latency of behavioral responses such as head-waving by damping and slowing muscular contractions. Thus, a complete understanding of the relationship between muscle activity and overt behavior will require an analysis of both the biomechanics and hoemodynamics of movement in Aplysia (see, for example, Kuenzi and Carew, 1988).

The relatively long phase lag that we observe between EMG in the LCMs and the head-waving response may have important implications for the cellular mechanisms of operant conditioning. For example, in order for operant conditioning to occur, an animal must be able to associate its own behavioral output with the reinforcement it receives. If Aplysia use peripheral feedback from their muscles to detect their own behavioral output, it may be that animals require a central mechanism to compensate for the long delay of feedback to the CNS from the periphery. Alternatively, such a compensatory mechanism might be unnecessary if the central activity giving rise to head-waving lasts long enough to have the opportunity to interact with input from reinforcement. A cellular analysis of operant conditioning will be required to distinguish between these and other alternatives. A more detailed analysis of the effector properties of the response system that expresses the conditioning is critical, both because it can suggest potentially important cellular mechanisms and because it can place important constraints on any mechanisms proposed to underlie the conditioning.

\section{LCMs provide a useful analog system for a cellular analysis of operant conditioning}

Head-waving behavior is a complex response that involves the coordinated action of a variety of different muscle groups in Aplysia. In order for a cellular analysis of operant conditioning to bc fcasiblc, it is important to identify a restricted group of experimentally accessible muscles that participate in head-waving behavior. Our results indicate that the activity of the LCMs is significantly related to the horizontal component of headwaving. Thus, these findings will facilitate a cellular analysis of operant conditioning because they permit us to focus on a restricted motor output pathway that is quantitatively related to naturally occurring head-waving behavior. Current studies in this restricted system include a biomechanical analysis of the role that the LCMs play in head-waving and the specification of the anatomical projections and functional role of identified motor neurons that innervate the LCMs (Kuenzi and Carew, 1988; and unpublished observations).

If the LCMs are to serve as an analog system for a cellular analysis of operant conditioning in Aplysia, a critical question is whether this restricted output system is itself capable of op- erant modification. We address this question in the companion paper that follows (Cook and Carew, 1989b).

\section{References}

Alkon, D. L. (1987) Memory Traces in the Brain, Cambridge U. P., Cambridge, England.

Bablanian, G. M., K. R. Weiss, and I. Kupfermann (1987) Motor control of the appetitive phasc of feeding behavior in Aplysia. Behav. Neural Biol. 48: 394-407.

Bailey, C. H., V. Castellucci, J. Koester, and E. R. Kandel (1979) Cellular studies of peripheral neurons in siphon skin of Aplysia californica. J. Neurophysiol. 42: 530-557.

Brace, R. C. (1977a) The functional anatomy of the mantle complex and columellar muscle of tectibranch molluscs (Gastropoda: Opisthobranchia), and its bearing on the evolution of opisthobranch organization. Phil. Trans. R. Soc. London [Biol.] 277: 1-56.

Brace, R. C. (1977b) Shell attachment and associated musculature in the notaspidea and anaspidea (Gastropoda: Opisthobranchia). Trans. Zool. Soc. London 34: 27-43.

Byrne, J. H. (1985) Neural and molecular mechanisms underlying information storage in Aplysia: Implications for learning and memory. Trends Neurosci. 8: 478-482.

Byrne, J. H. (1987) Cellular analysis of associative learning. Physiol. Rev. 67: 329-439.

Carew, T. J., and C. L. Sahley (1986) Invertebrate learning and memory: From behavior to molecules. Annu. Rev. Neurosci. 9: 435-487.

Carew, T. J., V. F. Castellucci, and E. R. Kandel (1971) An analysis of dishabituation and sensitization of the gill-withdrawal reflex in Aplysia. Int. J. Neurosci. 2: 79-98.

Carew, T. J., R. D. Hawkins, and E. R. Kandel (1983) Differential classical conditioning of a defensive withdrawal reflex in Aplysia californica. Science 219: 397-400.

Cobbs, J. S., and H. M. Pinsker (1982a) Role of bag cells in egg deposition of Aplysia brasiliana. I: Comparison of normal and elicited behaviors. J. Comp. Physiol. 147: 523-536.

Cobbs, J. S., and H. M. Pinsker (1982b) Role of bag cells in egg deposition of Aplysia brasiliana. II: Contribution of egg movement of elicited behaviors. J. Comp. Physiol. 147: 537-546.

Cook, D. G., and T. J. Carew (1986) Operant conditioning of headwaving in Aplysia. Proc. Natl. Acad. Sci. USA 83: 1120-1124.

Cook, D. G., and T. J. Carew (1988) Operant conditioning of identified neck muscles and individual motor neurons in Aplysia. Soc. Neurosci. Abstr. 14: 607.

Cook, D. G., and T. J. Carew (1989a) Operant conditioning of headwaving in Aplysia. II. Contingent modification of electromyographic activity in identified muscles. J. Neurosci. 9: 3107-3114.

Conk, D. G., and T. J. Carew (1989b) Operant conditioning of headwaving in Aplysia. III. Cellular analysis of possible reinforcement pathways. J. Neurosci. 9: 3115-3122.

Crow, T. (1985a) Conditioned modification of phototactic behavior in Hermissenda. I. Analysis of light intensity. J. Neurosci. 5: 209214.

Cruw, T. (1985b) Conditioned modification of phototactic behavior in Hermissenda. II. Differential adaptation of B photoreceptors. J. Neurosci. 5: 215-223.

Crow, T. J. (1988) Cellular and molecular analysis of associative behavioral changes in Hermissenda. Trends Neurosci. 11: 136-142.

Farley, J., and D. L. Alkon (1982) Associative neural and behavioral change in Hermissenda: Consequences of nervous system orientation for light- and pairing-specificity. J. Neurophysiol. 48: 785-807.

Ferguson, G. P., A. Ter Maat, D. W. Parsons, and H. M. Pinsker (1989a) Egg laying in Aplysia. I: Behavioral patterns and muscle activity of freely behaving animals after selectively elicited bag cell discharges. J. Comp. Physiol. 164: 835-847.

Ferguson, G. P., A. Ter Maat, and H. M. Pinsker (1989b) Egg laying in Aplysia. II: Organization of central and peripheral pathways for initiating neurosecretory activity and behavioral patterns. J. Comp. Physiol. 164: 849-857.

Fredman, S. M., and B. Jahan-Parwar (1983) Command neurons for locomotion of Aplysia. J. Neurophysiol. 49: 1092-1117.

Hawkins, R. D., T. W. Abrams, T. J. Carew, and E. R. Kandel (1983) A cellular mechanism of classical conditioning in Aplysia: Activitydependent amplification of presynaptic facilitation. Science 219:400405. 
Hawkins, R. D., G. A. Clark, and E. R. Kandel (1987) Cell biological studies of learning in simple vertebrate and invertebrate systems. In Handbook of Physiology, Sect. I. Higher Functions of the Nervous System, Vol. 6, F. Plum, ed., pp. 25-83, American Physiological Suciety, Bethesda, MD.

Hening, W. A., E. T. Walters, T. J. Carew, and E. R. Kandel (1979) Motorneuronal control of locomotion in Aplysia. Brain Res. 179: 231253.

Hochner, B., M. Klein, S. Schacher, and E. R. Kandel (1986a) Additional component in the cellular mechanism of presynaptic facilitation contributes to behavioral dishabituation in Aplysia. Proc. Natl. Acad. Sci. 83: 8794-8798.

Hochner, B., M. Klein, S. Schacher, and E. R. Kandel (1986b) Actionpotential duration and the modulation of transmitter release from the sensory neurons of Aplysia in presynaptic facilitation and behavioral sensitization. Proc. Natl. Acad. Sci. USA 83: 8410-8414.

Horowitz, L. M. (1974) Elements of Statistics for Psychology and Education, McGraw-Hill, New York.

Hoyle, G. (1982a) Cellular basis of operant-conditioning of leg position. In Conditioning: Representation of Involved Neural Functions, C. D. Woody, ed., pp. 197-211, Plenum, New York.

Hoyle, G. (1982b) Pacemaker change in learning paradigm. In Cellular Pacemakers, D. Carpenter, ed., pp. 3-25, Wiley, New York.

Jahan-Parwar, B., and S. M. Fredman (1978a) Control of pedal and parapodial movements in Aplysia. I. Proprioceptive and tactile reflexes. J. Neurophysiol. 41: 600-608.

Jahan-Parwar, B., and S. M. Fredman (1978b) Control of pedal and parapodial movements in Aplysia. II. Cerebral ganglion neurons. J. Neurophysiol. 41: 609-620.

Kandel, E. R., and J. H. Schwartz (1982) Molecular biology of learning: Modulation of transmitter release. Science 218: 433-443.

Kuenzi F. M., and T. J. Carew (1988) A biomechanical analysis of the head-waving response in Aplysia. Soc. Neurosci. Abstr. 14:1000.

Kupfermann, I. (1970) Stimulation of egg-laying by extracts of neuroendocrine cells (bag cells) of abdominal ganglion of Aplysia. J. Neurophysiol. 33: 877-881.

Kupfermann, I. (1974a) Feeding behavior in Aplysia: A simple system for the study of motivation. Behav. Biol. 10:1-26.

Kupfermann, I. (1974b) Dissociation of the appetitive and consummatory phases of feeding behavior in Aplysia: A lesion study. Behav. Biol. 10: 89-97.
Kupfermann, I., and T. J. Carew (1974) Behavioral patterns of Aplysia californica in its natural environment. Behav. Biol. 12: 317-337.

Kupfermann, I., T. J. Carew, and E. R. Kandel (1974) Local, reflex, and central commands controlling gill and siphon movements in Aplysia. J. Neurophysiol. 37: 996-1019.

Mackintosh, N. J. (1974) The Psychology of Animal Learning, Academic, London.

Marcus, E. A., T. G. Nolen, C. H. Rankin, and T. J. Carew (1988) Behavioral dissociation of dishabituation, sensitization, and inhibition of Aplysia. Science 241: 210-213.

Mpitsos, G. J., and K. Lukowiak (1986) Learning in gastropod molluscs. In The Mollusca: Neurobiology and Behavior, Vol. 8, A. O. D. Willows, ed., pp. 96-267, Academic Press, Orlando, FL.

Pinsker, H. M., and L. B. Eberly (1982) Whole-nerve cuff electrodes in neuroethological studies. J. Electrophysiol. Tech. 8: 88-101.

Preston, R. J., and R. M. Lee (1973) Feeding behavior in Aplysia californica: Role of chemical and tactile stimuli. J. Comp. Physiol. 82: 368-381.

Rescorla, R. A. (1988) Behavioral studies of Pavlovian conditioning. Annu. Rev. Neurosci. 11:329-352.

Teyke, T., and I. Kupfermann (1988) Analysis of directed head movements in response to food stimuli in Aplysia. Soc. Neurosci. Abstr. 14: 608 .

Walters, E. T., and J. H. Byrne (1983a) Associative conditioning of single sensory neurons suggests a cellular mechanism for learning. Science 219: 405-408.

Walters, E. T., and J. H. Byrne (1983b) Slow depolarization produced by associative conditioning of Aplysia sensory neurons may enhance $\mathrm{Ca}^{++}$entry. Brain Res. 280: 165-168.

Walters, E. T., J. H. Byrne, T. J. Carew, and E. R. Kandel (1983a) Mechanoafferent neurons innervating the tail of Aplysia. I. Response properties and synaptic connections. J. Neurophysiol. 50: 1522-1542.

Walters, E. T., J. H. Byrne, T. J. Carew, and E. R. Kandel (1983b) Mechanoafferent neurons innervating the tail of Aplysia. II. Modulation by sensitizing stimuli. J. Neurophysiol. 50: 1543-1559.

Woolacott, M. H., and G. Hoyle (1977) Neural events underlying learning in insects: Changes in pacemaker. Proc. R. Soc. London Ser. B 195: 395-415. 